\title{
Effect of plasma polarity on the synthesis of graphene quantum dots by atmospheric-pressure microplasmas
}

\author{
Thomas Orriere \\ E-mail: thomas.orriere@univ-poitiers.fr \\ Institut Pprime (CNRS UPR 3346-Universit de Poitiers-ENSMA), F-86962, \\ Chasseneuil Futuroscope, France
}

\section{Darwin Kurniawan}

Department of Chemical Engineering, National Taiwan University of Science and Technology, Taipei, 10607, Taiwan

\section{Yi-Chen Chang}

Department of Chemical Engineering, National Taiwan University of Science and Technology, Taipei, 10607, Taiwan

\section{David Z. Pai}

E-mail: david.pai@univ-poitiers.fr

Institut Pprime (CNRS UPR 3346-Universit de Poitiers-ENSMA), F-86962,

Chasseneuil Futuroscope, France

\section{Wei-Hung Chiang}

E-mail: whchiang@mail.ntust.edu.tw

Department of Chemical Engineering, National Taiwan University of Science and Technology, Taipei, 10607, Taiwan

\begin{abstract}
.
The aim of this study is to optimize the production of colloidal graphene quantum dots (GQD) in an aqueous solution containing sodium dodecyl sulfate (SDS) treated by an argon microplasma jet operated in open ambient air. The plasma has been investigated by optical emission spectroscopy and electrical measurements, and the produced GQDs have been studied by Raman spectroscopy, photoluminescence, UV-visible absorption, transmission electron microscopy and atomic force microscopy. We mainly focus on the influence of the polarity of the voltage applied to generate the microplasma. Although the deposited power is higher when using a positive polarity, the energy efficiency is also higher thanks to a faster synthesis rate. To understand the underlying mechanisms, we reproduced the experiments with the addition of $\mathrm{H}_{2} \mathrm{O}_{2}$ in the aqueous solution. Results show that the GQD synthesis operates in two steps with SDS fragmentation followed by an electrolysis-related process. We demonstrate that the positive polarity performs better due to higher fragmentation rate.
\end{abstract}




\section{Introduction}

As a zero-dimensional carbon nanomaterial, graphene quantum dots (GQDs) have gained enormous popularity owing to their tunable and stable photoluminescence properties, biocompatibility, and chemical stability. Numerous GQD-based applications have been reported, including bioimaging, chemical and biological sensing, drug delivery, catalysis, LEDs, and energy-related applications (Zheng et al. 2015, Yan et al. 2019, Shen et al. 2012, Kumar et al. 2020). The synthesis of GQDs is classified into top-down and bottom-up methods. The former involves exfoliation from bulk carbon materials into GQDs which is excellent for large scale production. However, it is difficult to obtain a well-controlled structure and requires the usage of toxic and hazardous chemicals, along with long reaction time and harsh conditions (Kumar et al. 2020, Pan et al. 2010, He et al. 2018, Valappil et al. 2017). Meanwhile, the latter provides ease of tunability though carbonization, pyrolysis or step-wise chemical reaction pathways, but usually suffers from low production yield, long reaction time, and tedious synthesis procedures (Kumar et al. 2020, Valappil et al. 2017, Dong et al. 2012). Among these methods, bottom-up synthesis atmospheric microplasma jets is promising because of its scalability and simplicity (Chiang et al. 2010). Usually, an aqueous solution containing the precursors is exposed to a plasma jet supplied with an inert gas such as argon or helium. The plasma is generated between the jet and the liquid by applying high voltage.

When the plasma is geometrically confined under the mm-scale, it obtains useful properties such as low energy consumption, low gas heating, stability and higher reactive species densities. This special class of discharge is called the microplasmas (Chiang et al. 2019). The ignited plasmas help accelerate chemical reactions and promote processes which are usually difficult to achieve (Chiang et al. 2019).

With these advantages, microplasmas have been exploited to synthesized GQDs or carbon dots (CDs) with tunable properties using simple carbon-containing precursors (Kurniawan \& Chiang 2020, Yang, Pai \& Chiang 2019, Wang et al. 2015, Joffrion et al. 2019, Ma et al. 2019). GQDs with a $4.9 \mathrm{~nm}$ size have been produced by treating an organosulfate aqueous solution by an atmospheric microplasma jet in ambient air (Yang, Pai \& Chiang 2019). DC negative voltage was applied to the jet. By adding $\mathrm{H}_{2} \mathrm{O}_{2}$, which is an electron scavenger (Rumbach et al. 2015), the authors showed that solvated electrons play a major part in GQD formation and the possible mechanism was discussed. During the synthesis, initially activated dodecanol molecules and related moieties were formed due to the SDS molecules interacting with the electrons and the radicals produced by the plasma. Thereafter, the activated species were cyclized and subsequently reconstructed to form nanographenelike structures, yielding to GQDs. However, faster production of carbon dots from ethylenediamine occurs when applying positive rather than negative DC voltage to the jet (Wang et al. 2015). In electrochemistry, the term "plasma anode" often refers to applying a positive polarity on the jet (as in (Wang et al. 2015)). In this paper, we use the term positive discharge (PD) when applying a positive voltage and negative discharge (ND) when applying a negative voltage to avoid any confusion with the plasma cathode or plasma anode. As pointed by Wang et al. (Wang et al. 2015), comparisons of PD and ND are rarely reported, even though in some cases the PD shows faster and more energy efficient nanoparticle synthesis. For silver nanoparticle synthesis, Shirai et al. (Shirai et al. 2014) showed a faster synthesis by using the ND 


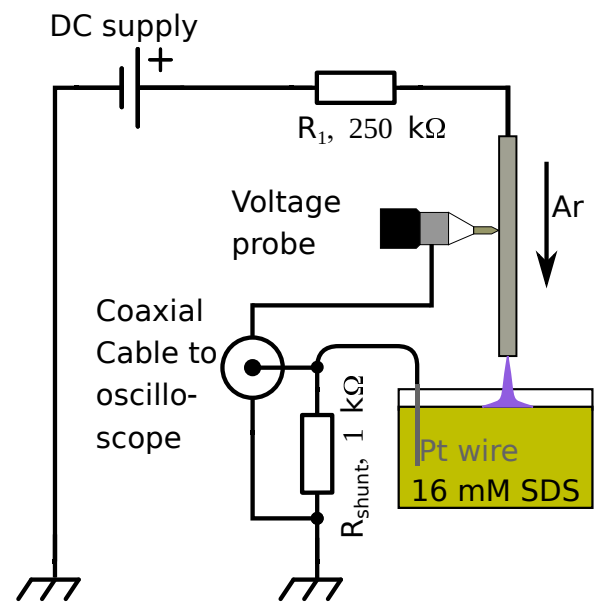

Figure 1: Experimental setup of the microplasma jet (PD). The DC power supply is reversed for the ND.

whereas gold nanoparticles were more easily produced by the PD.

The aim of this paper is to compare the production of GQDs from Sodium Dodecyl Sulfate (SDS) aqueous solution with PD and ND. In the PD experiment, the metal tube is the anode of the discharge. In the ND experiment, the solution is the anode. We used electrical measurements with passive probes and optical emission spectroscopy to monitor the deposited power and the emitted light from the discharge during the experiment. By examining photoluminescence and UV-visible absorption spectra of our samples, we show a faster production of the GQDs with PD. Additionally, the GQD structure was studied by employing transmission electron microscopy (TEM) and atomic force microscopy (AFM).

\section{Experimental setup}

The experimental setup is shown on figure 1. A DC power supply (Stanford Research Systems Model PS 350) is connected via a ballast resistor $R_{1}=250 \mathrm{k} \Omega$ to a capillary tube with an inner diameter of $180 \mu \mathrm{m}$. Argon gas was flow through the tube at a flow rate of $25 \mathrm{sccm}$. The $10 \mathrm{~mL}$ solution contained DI Water and SDS (Sigma Aldrich, CAS number 151-21-3) at a concentration of $16 \mathrm{mM}$. The $10 \mathrm{~mL}$ solution was placed into a $20 \mathrm{~mL}$ glass beaker and grounded via a shunt resistor $R_{\text {shunt }}=1 \mathrm{k} \Omega$ in series with a Pt electrode. For synthesis experiments, the grounded electrode was a DC plate immersed $1 \mathrm{~mm}$ in depth into the solution, but for the electrical measurements, we used a Pt wire with a similar cross-sectional area of immersion. The gap distance between the liquid and the tube was about $1 \mathrm{~mm}$. For the power supply, the voltage was set to $\pm 2 \mathrm{kV}$ while the current was maintained at $5 \mathrm{~mA}$ for both PD and ND. These are averaged values, but time-resolved voltage and current waveforms were recorded with an oscilloscope (Lecroy Waverunner 204Mxi $2 \mathrm{GHz}$ bandwidth) using a passive high voltage probe (Lecroy $400 \mathrm{MHz}$ bandwidth) and a low-inductance currentsensing resistor (Caddock), respectively. Transmission electron microscopy (TEM) measurements were performed using a field emission gun TEM (FEI TecnaiTM G2 F-20 S-TWIN) with an accelerating voltage of $200 \mathrm{kV}$. TEM samples were prepared 
from GQDs solution dry-casting onto formvar/carbon-coated copper grids (300 mesh, EM Resolutions Ltd.). Digital Micrograph 3.7 software was utilized to obtain the particles size distributions. The topographic heights of the synthesized GQDs were measured by an Atomic Force Microscope (Ardic Instrument P-100 AFM) in tapping mode. AFM samples were prepared from GQDs solution dry-casting onto a mica disc (9.9 mm diameter, Ted Pella Inc.). Ardic Instrument image processing software was used to obtain the particles height distributions.

\section{Results}

\subsection{Electrical characterization}

The voltage was measured at the steel capillary tube, representing the potential over the plasma and the solution. For both PD and ND, the current supply was maintained at $5 \mathrm{~mA}$ during at least 50 minutes and seemed stable to the naked eye and on the DC power supply current monitor. Occasionally the PD extinguished and needed to be reignited manually. We report on figure 2a an example of the time resolved voltage, current and power at $t=30 \mathrm{~min}$ after initiating the discharge, for both polarities. The main discrepancy between the the two discharges is that the current, voltage and power fluctuate more for the PD. The voltage oscillates mostly between 0.4 and 0.7 $\mathrm{kV}$, the current between 2 and $8 \mathrm{~mA}$ and the power between 0.7 and $4 \mathrm{~W}$. We also observed brief extinctions of the discharge where the current drops to zero and the voltage can rise up to $1.4 \mathrm{kV}$ as shown in figure $2 \mathrm{a}$ between $\mathrm{t}=7 \mu \mathrm{s}$ and $\mathrm{t}=13 \mu \mathrm{s}$. When the discharge reignites itself, current and power peaks reach amplitudes of 50 $\mathrm{mA}$ and $35 \mathrm{~mW}$ respectively. These strong fluctuations are limited by the discharge circuit including the DC power supply.

We recorded the electrical signals during time windows of $0.5 \mathrm{~ms}$ duration every $20 \mathrm{~s}$ to monitor the fluctuations over the course of synthesis. The standard deviation of the current, shown on figure $2 \mathrm{~b}$, increases with time up to values on the order of the averaged current for the PD, but never exceeds $1 \%$ of the average for the ND. The height of the solution changes with time. Therefore, we monitored this level and added DI water to maintain a gap distance of about $1 \mathrm{~mm}$. This issue is more severe in the PD case. As a result, the voltage slowly increases with experiment time and quickly decreases when DI water is added (figure $2 \mathrm{~b}$ ). The rise of the standard deviation indicates that the discharge evolves from a DC glow like discharge to a pulsed glow like regime and/or a self pulsing regime. The ND, however, is a true DC discharge. For the same gap distance, higher applied voltage is required for the PD to maintain the same current. Therefore, a higher total power is dissipated in the discharge and the liquid for the PD ( $2.5 \mathrm{~W})$ than for the ND $(1.8 \mathrm{~W})$.

\subsection{Discharge emission}

In this section, we describe the light emission of the ND and the PD. Imaging of the PD and ND enabled control of the solution level in the beaker. The PD is always brighter than the ND. The integrated emission intensities have been computed from the images of the ND and PD and are shown on figure $3 a$ relative to its initial value. The intensity of the ND decays significantly with time, unlike the PD.

The emission has also been monitored by optical emission spectroscopy (OES). As mentioned previously, the PD requires manual re-ignition at later times, probably 


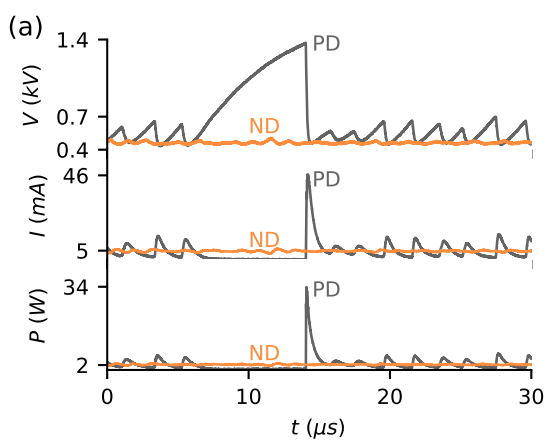

(a)

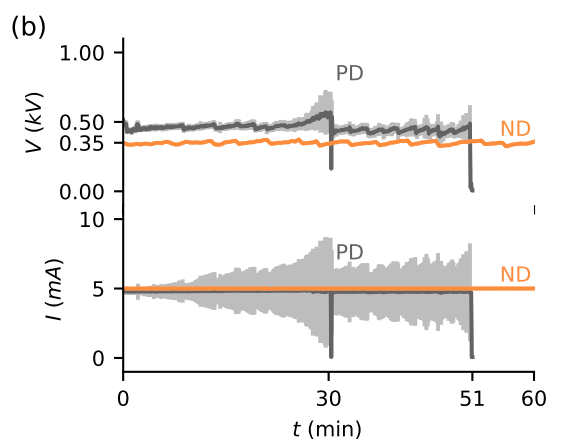

(b)

Figure 2: (a) Typical voltage, current and power waveforms for $t=30 \mathrm{~min}$ and (b) Evolution of the mean (darker shades) and the standard deviation (lighter shades) of the voltage $\mathrm{V}$ and the current $\mathrm{I}$ as a function of the experiment time.

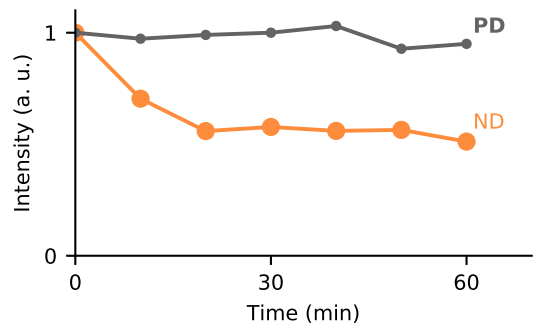

(a)

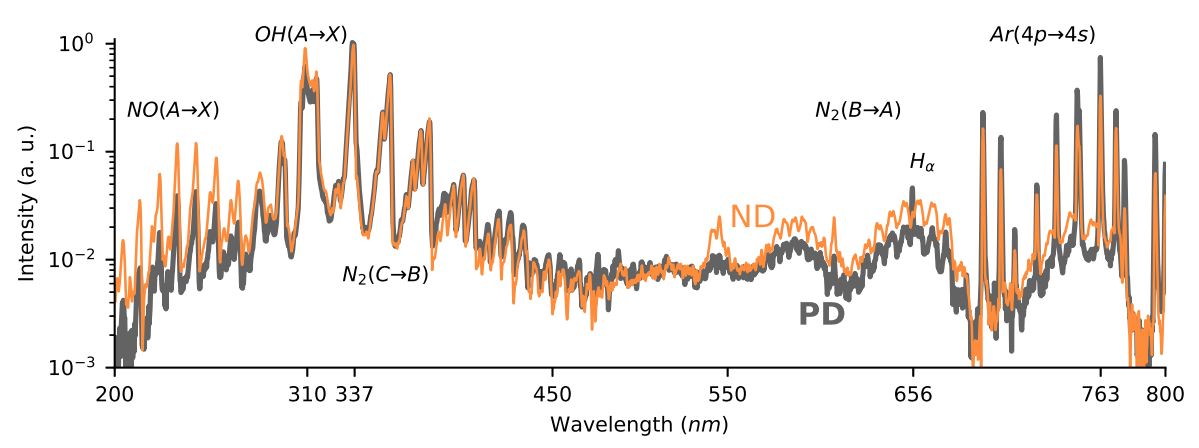

(b)

Figure 3: (a) Total light emission of the PD (grey) and the ND (orange), normalized to the intensity at $t=0 \mathrm{~s}$ and (b) time integrated spectra normalized by the intensity of the $N_{2}(C \rightarrow B)(0,0)$ band of the microplasma jet for both PD and ND.

when the solution evaporates or becomes too conductive. To mitigate this problem, for OES measurements $\mathrm{H}_{2} \mathrm{O}_{2}$ (an electron scavenger) was added in the solution at 
a concentration low enough $(0.005 \mathrm{mM})$ not to affect the UV-vis spectra. Timeintegrated spectra in both cases, shown in figure 3b, are largely similar but exhibit several discrepancies. For the PD, the atomic argon lines have higher relative intensities, and hydrogen atom balmer line $H_{\alpha}$ can be observed. On the other hand, the relative intensity of molecular bands such as $O H(A \rightarrow X), N O(A \rightarrow X)$ and $N_{2}(B \rightarrow A)$ are higher for the ND. For the ND, the total intensity as well as the individual intensities of the $N_{2}$ bands and the $A r$ lines start to decrease after 15 minutes. The emission spectra exhibits the same decay discrepancy as the integrated emission, shown on figure 3a. The decay is slower for the $\mathrm{PD}$, and the balance between the $A r$ and $N_{2}$ emission changes. The OES data were obtained without control of the gap distance, showing that the time evolution of discharge emission is robust. The discharge emission and the electrical characterization showed that the PD is unstable and needs to be reignited. Furthermore, the reignition is harder with the PD than for the ND. Increasing the resistance between the jet and the power supply can make ignition of the PD easier. Also, the addition of $\mathrm{H}_{2} \mathrm{O}_{2}$ stabilizes the discharge while $\mathrm{HCl}$ destabilizes it.

\subsection{GQDs properties}

We used UV-Vis absorbance to characterize the effect of polarity as well as the addition of $\mathrm{H}_{2} \mathrm{O}_{2}$ at a concentration of $0.05 \mathrm{mM}$ on GQD production for 1 hour of synthesis time. The absorbance spectra for PD and ND, shown on figure 4(a), both indicate similar profiles with strong UV absorption and a shoulder positioned between $250 \mathrm{~nm}$ and $300 \mathrm{~nm}$. These features are usually associated with the $\pi \rightarrow \pi^{*}$ and $n \rightarrow \pi^{*}$ transitions of aromatic $\mathrm{C}=\mathrm{C}$ structures. The highest production yield was obtained with the PD without $\mathrm{H}_{2} \mathrm{O}_{2}$. Both the polarity and the presence of $\mathrm{H}_{2} \mathrm{O}_{2}$ have an influence. The yield of the GQDs synthesized from both cases were quantified by drying the purified GQDs to obtain GQD powder. Then, different amounts of GQD powder were weighed and dissolved in $1 \mathrm{~mL}$ of DI water, and the absorbance of the solution with different GQD contents was measured using a UV-Vis spectrometer. A standard concentration calibration line was constructed by plotting the concentration versus absorbance, from which the GQD concentration can be quantified and the production yield can be estimated. The production yield of the ND is only $20.37 \%$ while the PD can offer almost 4 times higher, $79.32 \%$. The deposited energy per unit mass $\epsilon_{m}\left(J . g^{-1}\right)$ produced can be calculated by equation 1 with the averaged power $P(W)$ (see section 3.1), the synthesis time $T(s)$, the volume of the solution $V_{s}(L)$, the molarity $c_{s}(M)$ and the molar mass of $\operatorname{SDS} M_{S D S}\left(\mathrm{~g} \mathrm{~mol}^{-1}\right)$. The PD offers an efficiency almost three times higher $\left(246 \mathrm{~J}_{\mathrm{mg}} \mathrm{m}^{-1}\right.$ ) than the ND $\left(691 \mathrm{~J} . \mathrm{mg}^{-1}\right)$. The energy cost using our microplasma reactor appears low compared to graphene synthesis using an Ar plasma torch (Dato et al. 2008), where $250 \mathrm{~W}$ of microwave power produced $2 \mathrm{mg} / \mathrm{min}$ of carbon products, equivalent to an energy cost of 7500 $\mathrm{J} / \mathrm{mg}$.

$$
\epsilon_{m}=\frac{T \times P}{\text { yield } \times V_{s} \times c_{s} \times M_{S D S}}
$$

Raman spectra summarized on figure 4(b) confirmed the synthesis of GQDs. The three large peaks positioned at $1350 \mathrm{~cm}^{-1}, 1590 \mathrm{~cm}^{-1}$ and $2700 \mathrm{~cm}^{-1}$ could be assigned respectively to the D, G and 2D bands of graphene (Yang, Li, Lu, Yu, Huo, Man, Pan, Si, Jiang \& Zhang 2019, Kim et al. 2013). Also plotted on the figure is 


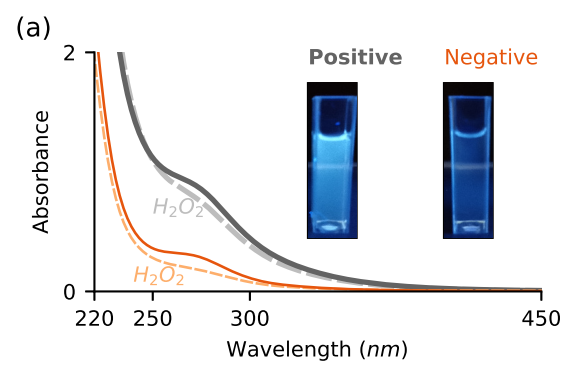

(a)

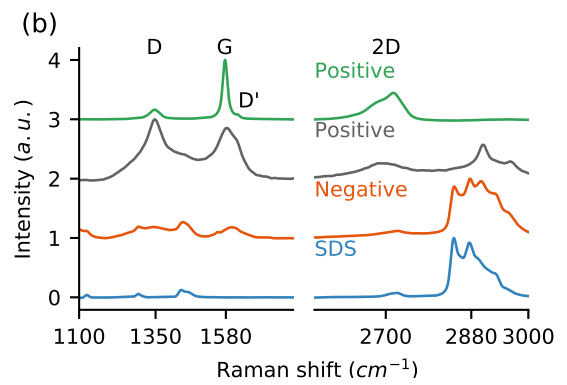

(b)

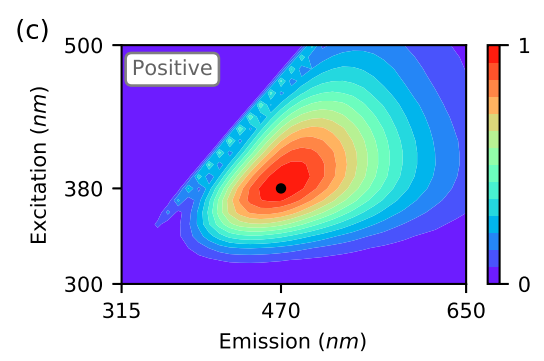

(c)

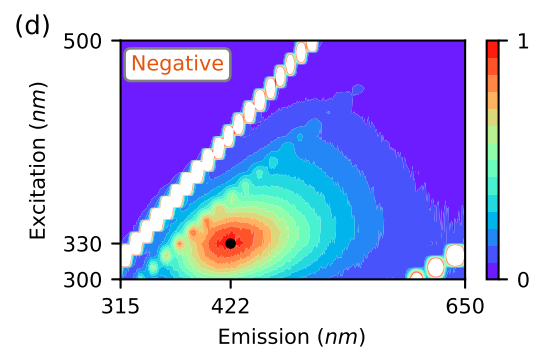

(d)

Figure 4: (a) Comparison of the UV/vis absorption spectra of the product obtained with the PD and the ND in pure SDS solution (darker shade) and with the addition of $\mathrm{H}_{2} \mathrm{O}_{2}$ (0.05 mM, dashed lighter shade). (b) Raman spectra of the SDS solution, as well as the products from ND and PD synthesis. (c) Normalized photoluminescence colormap of the product after synthesis by using the PD and (d) the ND. 
the Raman spectrum of the precursor (SDS). Therefore, the peaks positioned at 1290 $\mathrm{cm}^{-1}, 1440 \mathrm{~cm}^{-1}, 2840 \mathrm{~cm}^{-1}$ and $2880 \mathrm{~cm}^{-1}$ found in the spectra of the synthesized products belong to the precursor. For both PD and ND, the aliphatic hydrocarbon of SDS has been transformed into graphene-like structures. For the ND, the SDS remains on the sample while it is barely observable for the PD, indicating a higher conversion rate. Two types of spectra were identified from the sample produced by the PD. The first contains broad D, G and 2D peaks as observed with the ND (dark grey on figure $4(\mathrm{~b})$ ). The intensity ratio is $I_{D} / I_{G}=1.22$ compared to $I_{D} / I_{G}=1.27$ for the ND. The second, occurring more rarely, possesses sharp D and G bands (green on figure 4(b)). According to Ferrari et al.(Ferrari et al. 2006), the latter can be attributed to a structure of several layers of graphene due to the shoulder observable on the $2 \mathrm{D}$ peak at $2700 \mathrm{~cm}^{-1}$. The inhomogeneous result of the Raman data could be due to the sample preparation.

Representative photoluminescence (PL) spectra of the product from the PD and the ND, respectively illustrated by figures 4 (c) and (d), indicate a broadband emission when the samples were excited in the 300 to $500 \mathrm{~nm}$ range. The maximum PL intensity of the ND sample is found at excitation and emission wavelengths of $330 \mathrm{~nm}$ and $422 \mathrm{~nm}$, respectively. A similar result is obtained for the PD but the PL center is red shifted to $380 \mathrm{~nm}$ excitation and $470 \mathrm{~nm}$ emission, indicating larger produced GQDs compared to the ND experiment. The quantum yield of the as-produced GQDs was estimated by collecting the PL emission spectra of various GQD dilutions. Identical measurements were performed using a calibration standard of quinine sulfate in $0.5 \mathrm{M}$ $\mathrm{H}_{2} \mathrm{SO}_{4}$ with a known efficiency of $54 \%$. We estimate the quantum yield of the GQDs to be around $1 \%$, which is comparable to that of carbon-based dots prepared by plasma methods (Wang et al. 2015, Huang et al. 2015).

TEM measurements were performed to understand the morphologies of the GQDs synthesized from both PD and ND. Particle-like nanostructures with no aggregation nor amorphous coatings can be observed in the PD case, as shown in figure 5(a). In contrast, most of the particles in the ND case are aggregated and enveloped by amorphous coatings (figure 5(a)). We attribute this amorphous coating to unreacted SDS or some intermediate by-products that bond with the functional groups of GQDs. These observations strongly support our findings where the plasma is brighter in the PD case (figure 3), providing higher energy to break almost all of the SDS moieties to form GQDs, as supported by the Raman measurements in figure 4. The crystalline structure of the synthesized GQDs from both cases was confirmed from the fast Fourier transform (FFT) images (figure 5c). High-resolution TEM images reveal GQDs lattice spacings of 0.28 and $0.24 \mathrm{~nm}$, corresponding to the (002) and (1120) planes of graphite (Kumar et al. 2018) and graphene (Kurniawan \& Chiang 2020) respectively for the $\mathrm{PD}$ case, as depicted in figure 5(c). It is difficult to obtain an accurate lattice spacing for the ND case due to particle agglomeration. The corresponding size distribution histogram in figure $5(\mathrm{~d})$ shows average sizes of $3.79 \pm 0.73 \mathrm{~nm}$ and $4.32 \pm 0.81 \mathrm{~nm}$ based on standard normal distribution fitting over 40 and 25 different particles for PD and ND cases, respectively. The larger GQD size for the ND case may be due to particle aggregation, and the actual particle size can be much smaller since the maximum emission of PL is blue-shifted relative to the PD case. Moreover, Raman spectroscopy was also performed to study the product quality. In general, for carbon materials, the $\mathrm{D}$ and $\mathrm{G}$ bands are respectively assigned to the presence of disorders in carbon systems and to the inplane vibration of the CC bond, respectively (Dresselhaus et al. 2005). The Raman spectra of both the ND and PD cases show broad D and G peaks, similar 


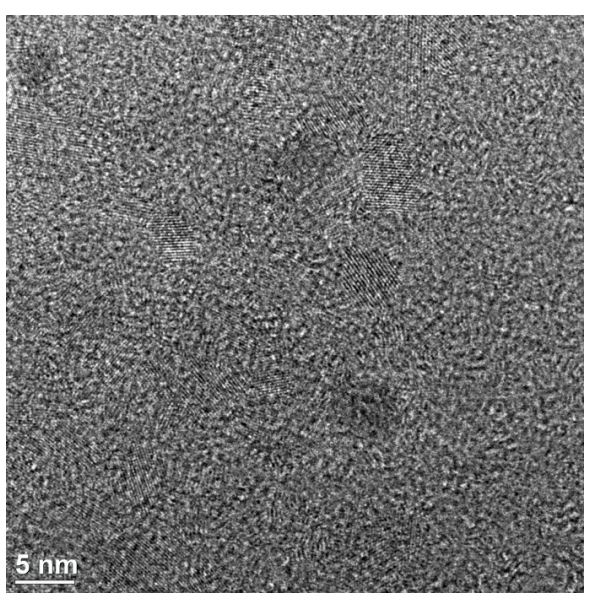

(a)

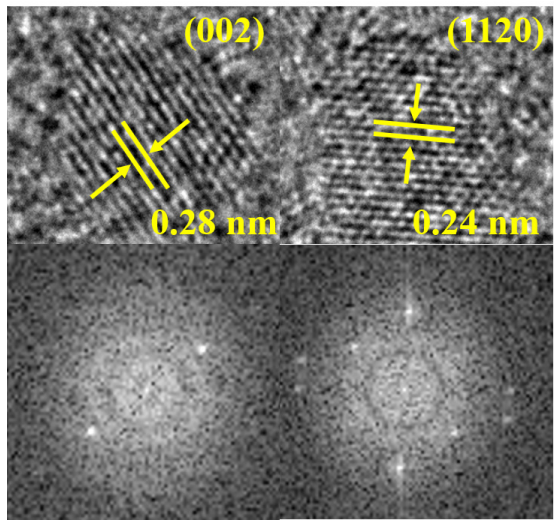

(c)

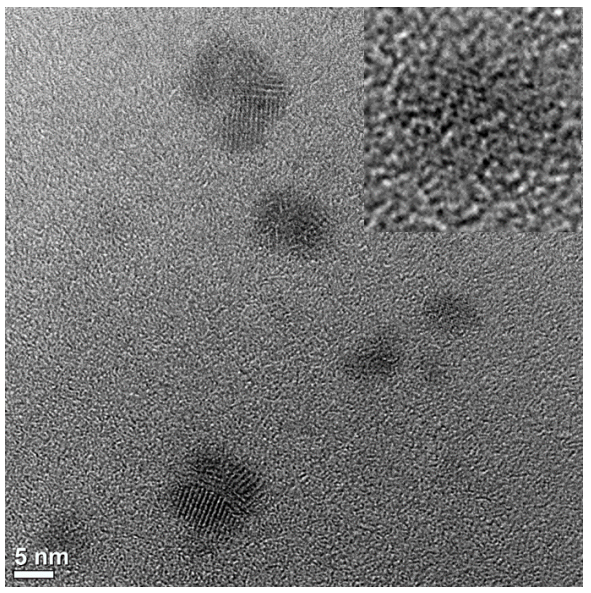

(b)

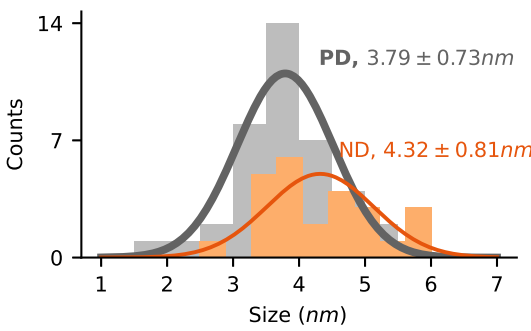

(d)

Figure 5: TEM images of the synthesized GQDs (a) by the PD (low resolution), (b) by the ND (low and high resolution) and (c) high resolution images of the PD product with the highlighted lattice spacing and corresponding fast Fourier transform. (d) The corresponding histogram obtained with 40 and 25 samples for the PD and the ND, respectively.

to those found by (Kim et al. 2013) for GQDs. However, the PD cases also show sharp peaks suggesting the presence of an additional species of GQD with higher crystallinity, similar to the Raman spectra of GQDs shown by (Pan et al. 2010, Peng et al. 2012, Zhuo et al. 2012). Generally, the value of the intensity ratio of D band to $\mathrm{G}$ band $\left(I_{D} / I_{G}\right)$ is used to estimate the degree of disorder and defects of carbon materials (Dresselhaus et al. 2005). The lower $I_{D} / I_{G}$ in the green curve suggests product with higher crystallinity or less defects are existed in the product. Overall, the TEM and Raman data suggest good quality of the GQD for PD case.

AFM was conducted to identify the topographic height of GQDs synthesized from both cases. The AFM images (figure 6(a) for the PD and figure 6(b)) indicate quite uniform GQDs heights have been synthesized by atmospheric microplasma. The 


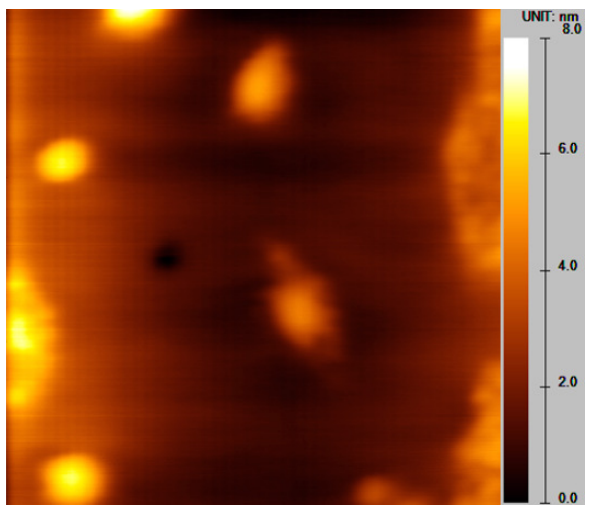

(a)

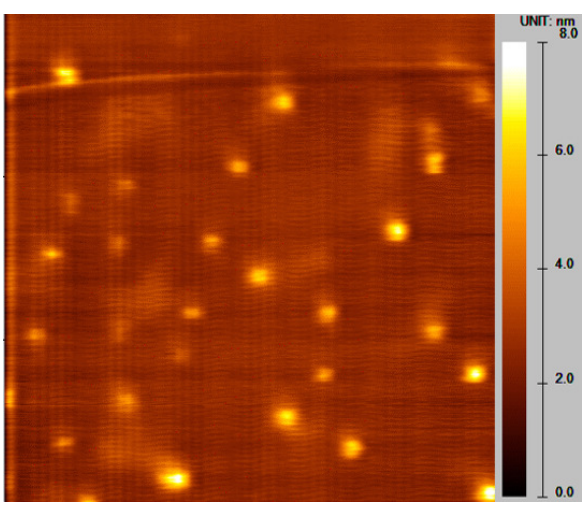

(b)

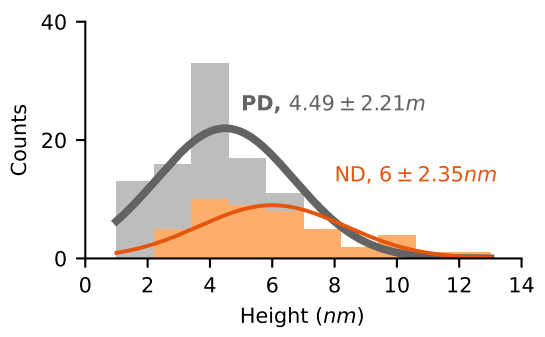

(c)

Figure 6: AFM images of the synthesized GQDs (a) by the PD and (b) by the ND with (c) the corresponding histogram obtained with 100 and 45 samples for the PD and the ND respectively.

corresponding histogram (figure 6(c)) was fitted with a normal distribution, yielding average heights of $4.49 \pm 2.21 \mathrm{~nm}$ and $6.00 \pm 2.35 \mathrm{~nm}$ to as-synthesized GQDs produced by PD and ND, respectively. These results indicate that the structure of synthesized GQDs for both cases consists of a few layers of graphene, consistent with our previous result (Kurniawan \& Chiang 2020). As with TEM measurements (figure 5(d)), it is also important to note that some aggregation and particle envelopment are observed in the AFM image for the ND case (figure 6c). Therefore, the actual value of the average topographic height of individual GQDs synthesized by ND may be shorter than in the PD case.

\subsection{Discussion}

In this section, we discuss all the results we presented in the previous sections in order to understand the mechanism for GQD production from the SDS precursor, particularly with regards to the voltage polarity. We discuss now all the results in order to understand the mechanism for GQD production from the SDS precursor particularly with regards to the voltage polarity. We first consider a straightforward explanation for the higher production by the PD discharge based on our electrical measurements in section 3.1. At equivalent gap distance, the power and the voltage 
applied on steel capillary are higher for the PD discharge. However, our UV absorption measurements showed an absorbance nearly 3 time higher for the PD than for the ND, whereas the power was only $40 \%$ higher. Therefore, increased power by itself cannot explain the higher production rate of the PD. Furthermore Yang et al.(Yang, Pai \& Chiang 2019) showed a linearly increasing production rate with increasing current or synthesis time using ND. This points to total charge injection as a key parameter in growth. Yet, in our case PD and ND production rates are quite different despite identical currents. However charge injection remains relevant, as demonstrated by the effect of adding $\mathrm{H}_{2} \mathrm{O}_{2}$. The suppression of GQD growth by using $\mathrm{H}_{2} \mathrm{O}_{2}$ as an electron scavenger for a ND has been demonstrated previously(Yang, Pai \& Chiang 2019).

In both cases $\mathrm{H}_{2} \mathrm{O}_{2}$ scavenging decreases GQD production by similar amounts according to the decrease in absorbance observed upon adding $\mathrm{H}_{2} \mathrm{O}_{2}$. Thus, even when high-energy plasma electrons are injected into the liquid (in the case of ND), their contribution to synthesis is in the form of charge rather than energy. Their energy is lost to some process that is not that critical to synthesis. However charge injection alone does not produce GQDs. We performed additional experiments for both PD and ND in which the plasma is replaced by a Pt wire just touching the solution, i.e. conventional electrolysis. Applying voltage to the $\mathrm{Pt}$ wire such that $5 \mathrm{~mA}$ ran through the solution did not result in GQD synthesis.

Next, we consider how the experiment evolves with time. The time-resolved emission of the ND during 60 minutes, presented previously, shows a sharp decrease of the emitted light from $N_{2}(C \rightarrow B)$ and the $A r$ lines, implying that the fluxes of the charged species, radicals, and photons also decrease with time. However, the input power, voltage and current are stable. It is also known that GQD production scales linearly with time and current (Yang, Pai \& Chiang 2019). As mentioned previously, current generation alone without plasma does not result in synthesis. Therefore, the variation of the plasma properties for the ND have a minor effect on the growth rate, but the plasma is needed to start the reaction by fragmenting or transforming the SDS with radicals, UV photons or charged species which are known to interact with the liquid (Bruggeman et al. 2016). The reaction is completed by a phenomenon dependent on charge injection, perhaps related to electrolysis.

The sustained strength of the PD over time compared to the ND would also imply more conversion of SDS. The Raman spectra indicate that the PD completely destroyed the SDS in the solution because no peaks from SDS remain after synthesis. The Raman spectrum obtained from the sample treated by the ND clearly shows some SDS peaks. The enhanced plasma properties of the PD may more effectively fragment SDS, as indicated by its more complete destruction, leading to the higher GQD production yield observed.

The PD may also create more favorable transport in the liquid. The PD may electrostatically attract the SDS to the area affected by the plasma and thus easily destroy the SDS. Visual inspection of bubble movement in the liquid suggests greater convection in $\mathrm{PD}$ than $\mathrm{ND}$, possibly due to electrohydrodynamic forces generated by the plasma and applied field. Shirai et al.(Shirai et al. 2014) suspected the influence of ion drift in the liquid, when using a dual plasma reactor (with both polarities) to produce either silver or gold nanoparticles. 


\section{Conclusion}

We have reported on the synthesis of GQDs from aqueous solution by a DC argon plasma jet at atmospheric pressure, focusing on the effect of the voltage polarity on the jet. The positive discharge (PD) is more effective for producing the GQDs, though less stable. PD stability can be improved by controlling the gap distance during the synthesis, and by adding $\mathrm{H}_{2} \mathrm{O}_{2}$ at concentrations as low as $0.005 \mathrm{mM}$. The light intensity decays during the first 30 minutes of synthesis with the ND, while this decay is rather slow for the PD. The first step for GQD synthesis is the fragmentation of the SDS, and the positive polarity has a greater ability for this purpose. Greater fragmentation of the PD may be due to more favorable plasma parameters (higher fluxes of photons, reactive species and ions, higher power and voltage) or indirectly via transport (electrohydrodynamic phenomenon, electrostatic drift). The second step appears to involve charge injection. The production yield increases with time and current yet is suppressed with the addition of $\mathrm{H}_{2} \mathrm{O}_{2}$, indicating that this electrolysis-related second step remains essential for both polarities. Finally, this study demonstrates that solvated electrons generated during the plasma interaction with liquid are not the only reactive species for GQD synthesis for the PD case because the electron flux for the PD is direct away from the liquid. Further experimental and modeling studies are necessary to provide more fundamental understanding.

\section{Acknowledgements}

This work was supported by the Partenariat Hubert Curien program Orchid (40938YL), the Ministry of Science and Technology of Taiwan (MOST Grant no. MOST 107-2628-E-011-002-MY3), National Taiwan University of Science and Technology (NTUST), the Agence Nationale de la Recherche program JCJC PLASMAFACE (ANR-15-CE06-0007-01), the Investissements dAvenir program LABEX INTERACTIFS (ANR-11-LABX-0017-01) of the French government, and the CPER-FEDER program of the Rgion Nouvelle Aquitaine.

The data that support the findings of this study are available from the corresponding author upon reasonable request.

\section{References}

Bruggeman P, Kushner M J, Locke B R, Gardeniers J G, Graham W, Graves D B, Hofman-Caris R, Maric D, Reid J P, Ceriani E et al. 2016 Plasma sources science and technology 25(5), 053002.

Chiang W H, Mariotti D, Sankaran R M, Eden J G \& Ostrikov K K 2019 Advanced Materials p. 1905508.

URL: https://onlinelibrary.wiley.com/doi/abs/10.1002/adma.201905508

Chiang W H, Richmonds C \& Sankaran R M 2010 Plasma Sources Science and Technology $19(3), 034011$.

Dato A, Radmilovic V, Lee Z, Phillips J \& Frenklach M 2008 Nano letters 8(7), 2012-2016.

Dong Y, Shao J, Chen C, Li H, Wang R, Chi Y, Lin X \& Chen G 2012 Carbon 50(12), 4738-4743.

Dresselhaus M S, Dresselhaus G, Saito R \& Jorio A 2005 Physics reports 409(2), 47-99.

Ferrari A C, Meyer J, Scardaci V, Casiraghi C, Lazzeri M, Mauri F, Piscanec S, Jiang D, Novoselov K, Roth S et al. 2006 Physical review letters 97(18), 187401.

He M, Guo X, Huang J, Shen H, Zeng Q \& Wang L 2018 Carbon 140, 508-520.

Huang X, Li Y, Zhong X, Rider A E \& Ostrikov K 2015 Plasma Processes and Polymers 12(1), 59-65.

Joffrion J B, Clower W \& Wilson C G 2019 Nano-Structures 85 Nano-Objects 19, 100341.

Kim S, Hee Shin D, Oh Kim C, Seok Kang S, Sin Joo S, Choi S H, Won Hwang S \& Sone C 2013 Applied Physics Letters 102(5), 053108. 
Kumar S, Aziz S T, Girshevitz O \& Nessim G D 2018 The Journal of Physical Chemistry C 122(4), 2343-2349.

Kumar Y R, Deshmukh K, Sadasivuni K K \& Pasha S K 2020 RSC Advances 10(40), 23861-23898. Kurniawan D \& Chiang W H 2020 Carbon .

Ma X, Li S, Hessel V, Lin L, Meskers S \& Gallucci F 2019 Chemical Engineering and ProcessingProcess Intensification 140, 29-35.

Pan D, Zhang J, Li Z \& Wu M 2010 Advanced materials 22(6), 734-738.

Peng J, Gao W, Gupta B K, Liu Z, Romero-Aburto R, Ge L, Song L, Alemany L B, Zhan X, Gao G et al. 2012 Nano letters 12(2), 844-849.

Rumbach P, Bartels D M, Sankaran R M \& Go D B 2015 Nature communications 6(1), 1-7.

Shen J, Zhu Y, Yang X \& Li C 2012 Chemical communications 48(31), 3686-3699.

Shirai N, Uchida S \& Tochikubo F 2014 Japanese Journal of Applied Physics 53(4), 046202.

Valappil M O, Pillai V K \& Alwarappan S 2017 Applied Materials Today 9, 350-371.

Wang Z, Lu Y, Yuan H, Ren Z, Xu C \& Chen J 2015 Nanoscale 7, 20743-20748. URL: http://dx.doi.org/10.1039/C5NR05804J

Yan Y, Gong J, Chen J, Zeng Z, Huang W, Pu K, Liu J \& Chen P 2019 Advanced Materials 31(21), 1808283.

Yang J S, Pai D Z \& Chiang W H 2019 Carbon 153, 315-319.

Yang W, Li Z, Lu Z, Yu J, Huo Y, Man B, Pan J, Si H, Jiang S \& Zhang C 2019 Optics Express $\mathbf{2 7}(3), 3000-3013$.

Zheng X T, Ananthanarayanan A, Luo K Q \& Chen P 2015 Small 11(14), 1620-1636.

Zhuo S, Shao M \& Lee S T 2012 ACS nano 6(2), 1059-1064. 